\title{
Prediction of GPI-anchored proteins with pointer neural networks
}

Gíslason, Magnús Halldór; Nielsen, Henrik; Almagro Armenteros, Jose Juan; Johansen, Alexander Rosenberg

\section{Published in:}

Current Research in Biotechnology

Link to article, DOI:

10.1016/j.crbiot.2021.01.001

Publication date:

2021

Document Version

Publisher's PDF, also known as Version of record

Link back to DTU Orbit

Citation (APA):

Gíslason, M. H., Nielsen, H., Almagro Armenteros, J. J., \& Johansen, A. R. (2021). Prediction of GPI-anchored proteins with pointer neural networks. Current Research in Biotechnology, 3, 6-13.

https://doi.org/10.1016/j.crbiot.2021.01.001

\section{General rights}

Copyright and moral rights for the publications made accessible in the public portal are retained by the authors and/or other copyright owners and it is a condition of accessing publications that users recognise and abide by the legal requirements associated with these rights.

- Users may download and print one copy of any publication from the public portal for the purpose of private study or research.

- You may not further distribute the material or use it for any profit-making activity or commercial gain

- You may freely distribute the URL identifying the publication in the public portal

If you believe that this document breaches copyright please contact us providing details, and we will remove access to the work immediately and investigate your claim. 


\title{
Prediction of GPI-anchored proteins with pointer neural networks
}

\author{
Magnús Halldór Gíslason ${ }^{\mathrm{a}, \mathrm{b}, \mathrm{e}}$, Henrik Nielsen ${ }^{\mathrm{a}, *}$, José Juan Almagro Armenteros ${ }^{\mathrm{a}, \mathrm{d}}$, \\ Alexander Rosenberg Johansen ${ }^{\mathrm{b}, \mathrm{c}}$ \\ ${ }^{a}$ Section for Bioinformatics, Department of Health Technology, Technical University of Denmark, Kgs. Lyngby 2800, Denmark \\ ${ }^{\mathrm{b}}$ Department of Applied Mathematics and Computer Science, Technical University of Denmark, Kgs. Lyngby 2800, Denmark \\ ${ }^{\mathrm{c}}$ Department of Computer Science, Stanford University, Stanford, CA 94305, USA \\ ${ }^{\mathrm{d}}$ Novo Nordisk Foundation Center for Protein Research, Faculty of Health and Medical Sciences, University of Copenhagen, Copenhagen 2200, Denmark \\ ${ }^{\mathrm{e}}$ Center for Genomic Medicine, Rigshospitalet, Copenhagen 2100, Denmark
}

\section{A R T I C L E I N F O}

\section{Keywords:}

Glycosylphosphatidylinositol

Lipid anchored proteins

Post-translational modification

Protein sorting

Prediction

Neural networks

\begin{abstract}
A B S T R A C T
GPI-anchors constitute a very important post-translational modification, linking many proteins to the outer face of the plasma membrane in eukaryotic cells. Since experimental validation of GPI-anchoring signals is slow and costly, computational approaches for predicting them from amino acid sequences are needed. However, the most recent GPI predictor is more than a decade old and considerable progress has been made in machine learning since then. We present a new dataset and a novel method, NetGPI, for GPI signal prediction. NetGPI is based on recurrent neural networks, incorporating an attention mechanism that simultaneously detects GPIanchoring signals and points out the location of their $\omega$-sites. The performance of NetGPI is superior to existing methods with regards to discrimination between GPI-anchored proteins and other secretory proteins and approximate ( \pm 1 position) placement of the $\omega$-site.

NetGPI is available at: https://services.healthtech.dtu.dk/service.php?NetGPI.

The code repository is available at: https://github.com/mhgislason/netgpi-1.1.
\end{abstract}

\section{Introduction}

Some of the proteins that follow the secretory pathway are bound to the membrane of eukaryotic cells by specific mechanisms. One of these mechanisms is a post-translational modification where a glycosylphosphatidylinositol (GPI) anchor is attached to the protein. The identification of proteins that undergo this modification is of high interest due to the diversity of functions that they perform. GPIanchored proteins are essential in the development of fungi and animal cells (Brul et al., 1997; Kawagoe et al., 1996). They are also involved in certain diseases such as paroxysmal nocturnal haemoglobinuria, an acquired haematopoietic stem-cell disorder (Takeda et al., 1993), and in the defense mechanisms of various protozoan parasites such as Leishmania and Trypanosoma (Masterson et al., 1990). Consequently, the development of computational tools that are able to detect proteins with this modification is of high impact on the research of eukaryotic cell biology (Mayor and Riezman, 2004).

GPI-anchored proteins have two signals in their primary sequence: an $\mathrm{N}$-terminal sequence for endoplasmic reticulum targeting (signal peptide) and a C-terminal signal sequence directing the attachment of the GPI-anchor. This attachment is carried out by a GPI transamidase which recognizes the C-terminal signal sequence and cleaves the peptide bond at the GPI-anchor attachment site, known as the $\omega$ site. This cleavage creates a covalent bond between the GPI and the C-terminus of the cleaved protein, allowing the protein to remain tethered to the membrane (Orlean and Menon, 2007). The transamidase is a multi-subunit complex; in mammals, it comprises the proteins PIGK, GPAA1, PIGS, PIGT, and PIGU. Among these, PIGK is the protease that cleaves off the propeptide, while GPAA1 seems to catalyse the formation of an amide bond between the $\omega$ amino acid and the GPI moiety (Kinoshita, 2020). The GPI modification is not in a strict sense reversible in vivo, since it involves the cleavage of the propeptide, but some GPI-anchored proteins are subsequently shed from the membrane by enzymatic cleavage of the GPI and thus converted into secretory proteins (Kinoshita, 2020).

C-terminal signal sequences are generally composed of five regions, which are determined by the amino acids before the $\omega$ site ( $\omega$-minus) and after ( $\omega$-plus). The five regions are: a stretch of polar amino acids that form a flexible linker region ( $\omega-10$ to $\omega-1)$; the $\omega$ site amino acid; the $\omega+2$ amino acid, a restrictive position with mostly G, A, or S; a spacer region of moderately charged amino acids $(\omega+3$ to $\omega+9$ or more), and a stretch of hydrophobic amino acids starting approximately at $\omega+10$ (Orlean and Menon, 2007).

\footnotetext{
* Corresponding author.

E-mail address: henni@dtu.dk (H. Nielsen).
} 
In order to detect proteins that carry this signal, experimental assays are required. Such experiments are generally low throughput and costly, which has resulted in a low amount of experimentally annotated GPI-anchored proteins. To overcome this limitation, fast computational methods that can approximate the experimentally validated process are needed. For this purpose, current machine learning methods exist for predicting GPI-anchors (Fankhauser and Mäser, 2005; Eisenhaber et al., 1999; Pierleoni et al., 2008). However, these methods were developed more than a decade ago and do not utilize recent progress in machine learning methods nor access to new data sources. Deep learning methods, such as recurrent neural networks (RNN) (Graves, 2012), have recently proven effective at protein prediction tasks (Jurtz et al., 2017). However, deep learning requires large amounts of annotated samples to generalize well (LeCun et al., 2015).

In this paper we present a new tool for detecting GPI-anchored proteins and determining the position of the $\omega$-site using recurrent neural networks. To overcome the low amounts of experimentally validated data we build a new dataset composed of both experimentally annotated and predicted GPI anchored proteins. To benchmark our method against previous methods, we only consider experimentally annotated samples. Regardless, our method achieves state-of-the-art performance on the GPI-anchor prediction task. Moreover, we show that the model learns biologically meaningful characteristics.

\subsection{Related works}

Initial work on predicting the presence of GPI-anchors and the $\omega$ site was published by Eisenhaber et al. (1999). This work, known as the Big-П Predictor, details a method that evaluates amino acid type preferences at positions near a potential $\omega$-site as well as the concordance with general physical properties encoded in multi-residue correlation within the motif sequence (Eisenhaber et al., 1999). Big-П provides kingdom-specific predictions as it was trained on metazoan, protozoan, fungi (Eisenhaber et al., 2004), and plant (Eisenhaber et al., 2003) proteins separately.

Fankhauser and Mäser (2005) presented a neural network based prediction tool called KohGPI/GPI-SOM. GPI-SOM utilizes a Kohonen Self Organizing Map structure, which takes as input the average position of a given amino acid relative to its proximity to the C-terminal, the hydrophobicity of the amino acid at $22 \mathrm{C}$-terminal positions, and 2 units representing the quality of the presumed $\omega$-site and its position. Both GPI-SOM and Big- $\Pi$ utilize an external signal peptide predictor known as SignalP (Armenteros et al., 2019) to preselect proteins.

FragAnchor was published by Poisson et al. (2007). FragAnchor uses a feed-forward neural network model to detect potential GPIanchoring signal sequences and a Hidden Markov Model (HMM) to quantify the prediction confidence and to estimate the position of the $\omega$-site, the spacer region and the hydrophobic tail. Like the previous two methods, FragAnchor relies on external evidence for the signal peptide and only regards the last $50 \mathrm{C}$-terminal amino acids. Unfortunately the prediction tool is no longer available online.

In 2008 Pierleoni, Martelli \& Casadio published Pred-GPI, a GPIanchor predictor using a Support Vector Machine (SVM) for the GPIanchoring signal discrimination and an HMM to predict the position of the $\omega$-site (Pierleoni et al., 2008). The HMM has 46 states with varying probabilities for amino acids and the potential $\omega$-site assigned to the 26th state. The SVM takes as input the negative log-likelihood computed by the HMM as well as 82 features intended to describe the overall composition of the sequence, the features of the $\mathrm{N}$ terminal regions comprising the signal peptide, and the features of the C-terminal regions containing the cleaved GPI-anchor signal. Pred-GPI supplies two different variants: one model where the potential $\omega$-site is restricted to be one of Cysteine, Aspartic acid, Glycine, Asparagine, and Serine - this approach they refer to as the conservative model - and a non-conservative variant which has no such restric- tion. Unlike the other three methods, Pred-GPI does not rely on an external signal peptide predictor, such as SignalP.

\section{Materials and methods}

\subsection{Dataset}

All data used in this project are extracted from the UniProt database, release 2019_02 (UniProt Consortium, 2014). The dataset construction follows two main steps: data gathering and homology partitioning. First, we select eukaryotic proteins with experimental (ECO:0000269) and non-experimental evidence of being GPIanchored and eukaryotic proteins with experimental evidence of being secreted, giving a total of 3618 proteins. All proteins are truncated to the last 100 amino acid positions. This is because our method does not include the prediction of the signal peptide and it is assumed that all relevant sequence information resides in or near the C-terminal positions. Instead, it relies on experimental evidence for the signal peptide or signal peptide prediction tools, such as SignalP (Armenteros et al., 2019). After truncation we remove exact duplicates, leaving 3567 unique sequences. Of the 3567 , there are 981 assumed to be GPIanchored. Out of the 981 there are 161 with experimental evidence for the GPI-anchoring signal of which 50 also have experimental evidence for the $\omega$-site, where the GPI-anchor would be attached. The remaining 820 have non-experimental evidence for the presence of a GPI-anchoring signal. This leaves 2586 without any evidence for the presence of a GPI-anchoring signal, which are assumed to be non GPI-anchored. The samples are also labelled by kingdom taxonomy: animal, fungi, plant, or other. The dataset composition is fully detailed in Table 1.

In order to measure a fair and unbiased estimate of the performance of a sequence-based prediction tool, it is essential to avoid homology between training and test sequences. This can be done by homology reduction or homology partitioning. In homology reduction, sequences are removed until no pair of sequences are more closely related than a specified threshold (Hobohm et al., 1992), while homology partitioning is the separation of a set of sequences into subsets, such that all sequences within each subset are less closely related than the threshold to sequences in other subsets. Commonly used clustering tools, such as CD-HIT (Li and Godzik, 2006), MMseqs 2 (Steinegger and Söding, 2017) and BLASTCLUST (Dondoshansky and Wolf, 2019), provide fast homology separation, where all sequences within each cluster are homologous to one another. However, these tools only ensure that a certain representative sequence in each cluster is less closely related than the threshold to sequences in other clusters; they do not guarantee that other members of the cluster follow the same criterion. They can therefore not be used for homology partitioning.

To homology partition the dataset, we therefore follow a four phase procedure, where we define percent identity of $30 \%$ as the threshold. First, we obtain global alignments using the program ggsearch36, which is a part of the FASTA package (Pearson and Lipman, 1988). The program implements the Needleman-Wunsch algorithm for global alignments (Needleman and Wunsch, 1970). We set the program's $E$ parameter, which is the expectation value threshold, to be larger than the dataset size, in order to include all pairwise comparisons in the output. We use the default output format, which calculates percent identity with the length of the alignment as the denominator. In the second phase, we cluster the pairwise percent identities, using restricted single-linkage clustering. As the end-goal is to partition the dataset into five comparable subsets, the clustering procedure is restricted such that no single cluster is allowed to have more than $\frac{981}{5}$ samples labelled GPI-anchored or $\frac{2586}{5}$ samples labelled non GPIanchored. In the third phase, the clusters are grouped together into five partitions, such that the number of GPI-anchored and non GPIanchored samples is comparable across all partitions. In the final 
Table 1

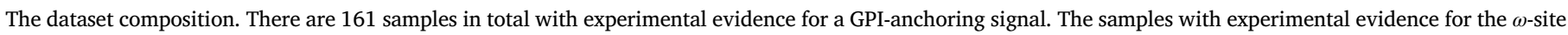
also have experimental evidence for a GPI-anchoring signal, here however, they are presented separately

\begin{tabular}{|c|c|c|c|c|c|}
\hline Kingdom & $\begin{array}{l}\text { Exp. ev. } \\
\omega \text {-site }\end{array}$ & $\begin{array}{l}\text { Exp. ev. } \\
\text { GPI-anchor }\end{array}$ & $\begin{array}{l}\text { Non-exp. ev. } \\
\text { GPI-anchor }\end{array}$ & $\begin{array}{l}\text { No ev. } \\
\text { for GPI-anchor }\end{array}$ & Total \\
\hline Animal & 25 & 72 & 417 & 2074 & 2588 \\
\hline Fungi & 7 & 27 & 220 & 336 & 590 \\
\hline Other & 5 & 2 & 77 & 19 & 103 \\
\hline Plant & 13 & 10 & 106 & 157 & 286 \\
\hline All & 50 & 111 & 820 & 2586 & 3567 \\
\hline
\end{tabular}

Abbreviation: Exp. ev. = Experimental evidence.

phase, samples are removed until no percent identity above $30 \%$ is found between samples in different partitions. During the removal phase, samples can be moved between partitions, as an attempt to reduce the number of samples removed. The composition of the final partitions can be seen in Table 2 and in the supplementary files. The final dataset contains 966 proteins labelled GPI-anchored and 2573 labelled non GPI-anchored, for a total of 3539 . Out of the 28 removed, one has experimental evidence for a GPI-anchoring signal sequence. All 50 samples with an experimentally verified $\omega$-site are retained.

\subsection{Objective}

The objective of GPI prediction is to decide whether a GPI signal is present and, if present, to determine the position of the $\omega$-site in a protein sequence. We combine these two tasks by reducing them to the single task of maximizing the probability of a position in a sequence. To achieve this, we add a placeholder to the end of the protein sequence which serves as an indicator for the absence of a GPIanchoring signal. Thus, we formally define the objective as maximizing the probability of a position in $\widehat{D}$, which is known as pointing (Vinyals et al., 2015).

$$
\begin{array}{r}
\max _{\theta} P_{\theta}\left(C_{i} \mid \widehat{D}\right) \\
\widehat{D}=[D, \mathrm{z}]
\end{array}
$$

Where $D \in \Sigma^{T-1}$ is an amino acid sequence and $\Sigma$ is a dictionary of the twenty common amino acids as well as the token $\mathrm{X}$, which represents any other encountered amino acid symbol. We only consider the last 100 amino acids in the protein sequence, such that the length $T-1 \leqslant 100$. If the sequence does not contain an $\omega$-site we maximize the probability of the protein being non GPI-anchored. Inspired by work in natural language processing (Merity et al., 2016; McCann et al., 2018), we represent the lack of an $\omega$-site by maximizing the placeholder position known as the sentinel, $z$, at the end of the amino acid sequence. This results in $\widehat{D} \in \hat{\Sigma}^{T}$ where $\hat{\Sigma}=\Sigma \cup\{z\}$. $C_{i}$ then corresponds to a position in $\widehat{D}$.

To parameterize the conditional probability distribution $P_{\theta}$ we use a neural network architecture known as the Long-Short Term Memory (LSTM) Cell (Hochreiter and Schmidhuber, 1997) and distributed representations of the amino acids (Mikolov et al., 2013) as shown in Eq. 3 ,

$$
\begin{array}{cc}
z_{i} & =\operatorname{embedding}\left(\hat{D}_{i}\right) \\
h & =\operatorname{LSTM}(\mathbf{z}) \\
g_{i} & =\tanh \left(h_{i} W\right) \\
P\left(C_{i} \mid \hat{D}\right)_{\theta}=\operatorname{softmax}(g V)_{i} & =\frac{\exp \left(g_{i} V\right)}{\sum_{j=0}^{T} \exp \left(g_{j} V\right)}
\end{array}
$$

where embedding $: \hat{\Sigma} \rightarrow \mathbb{R}^{d}$ transforms each amino acid into a distributed representation of real numbers using a linear trainable weight of size $d$ and $i, j \in \mathbb{N} \leqslant T$ are indexes of the protein sequence including the sentinel position. The LSTM is a non-linear transformation of a sequence of real values. It uses trainable recurrent units to distribute sequential information across the protein sequence, LSTM : $\mathbb{R}^{T \times d} \rightarrow \mathbb{R}^{T \times d^{\prime}}$, where $d^{\prime}$ is the output size of the LSTM. As we use a bidirectional LSTM (Schuster and Paliwal, 1997) we end up with two hidden representations of size $d^{\prime}$. To get the probability over the sequence we project the output of every position to a logit, $g_{i} V \in \mathbb{R}$, followed by a softmax : $\mathbb{R}^{T} \rightarrow[0,1]^{T}$ that normalizes the logits into a probability distribution over the sequence. To create the logits we use a two layer feed forward neural network on top of the LSTM hidden states, $h \in \mathbb{R}^{T \times 2 d^{\prime}}$, with a tanh activation function, $W \in \mathbb{R}^{2 d^{\prime} \times d^{\prime \prime}}$, and $V \in \mathbb{R}^{d^{\prime \prime}}$. This usage of softmax over a sequence is a modification of attention where the interaction size $d^{\prime \prime}$ of $g V$ is the attention hidden representation size. This modification of attention is known as a pointer network (Vinyals et al., 2015).

The embedding, LSTM, $W$, and $V$ are all trainable with stochastic gradient descent using back-propagation through time (Werbos, 1990). We have visualized our model in Fig. 1.

\subsection{Model details}

In order to measure the performance of our method on the entire dataset in an unbiased way, we use fivefold nested cross-validation. For each partition, the four other partitions are cross-validated where three partitions are used to train a model and one to validate the performance, i.e. to select the optimal hyperparameters and the point at which to stop the training. In all, 20 models are trained. The performances of different combinations of hyperparameters are compared

\begin{tabular}{|c|c|c|c|c|c|c|c|c|}
\hline Partition: & 0 & 1 & 2 & 3 & 4 & Label & Mean & Samples \\
\hline & 188 & 189 & 225 & 169 & 195 & Anchored & 193.2 & 966 \\
\hline & 488 & 484 & 484 & 629 & 488 & Not anchored & 514.6 & 2573 \\
\hline
\end{tabular}
using the average performance of the four models in the inner loop of the nested cross-validation. The SIGOPT platform is used for model

Table 2

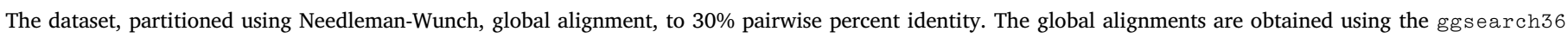
program, provided with the FASTA package. 


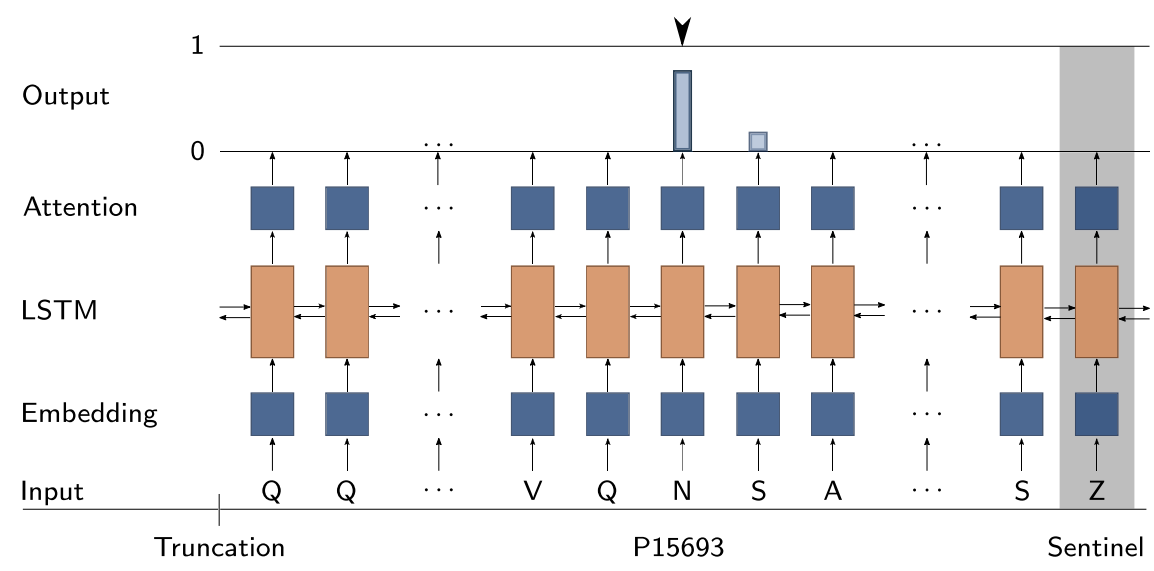

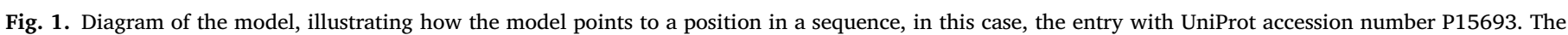

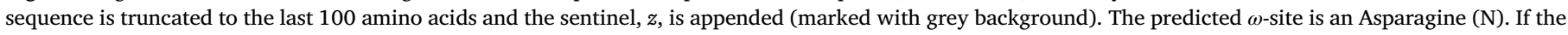
position with highest likelihood had been the sentinel position, then the protein would have been predicted as non GPI-anchored.

selection (Clark and Hayes, 2019). The SIGOPT optimization engine provides suggestions for hyperparameters based on inference from the performance of other hyperparameter combinations, measured with any real valued metric. The user supplies the values and specifies whether the value should be minimized or maximized. Each of the five sets of four models is trained with at least 200 hyperparameter combinations. The hyperparameters to tune are: The size of the distributed representation $(d)$, the LSTM cell hidden representation $\left(d^{\prime}\right)$, the number of LSTM layers, the LSTM dropout, the attention hidden representation $\left(d^{\prime \prime}\right)$, the batch size, and the optimizer's learning rate, learning rate decay and weight decay. Each epoch, after the first, the learning rate is updated by multiplying it with the learning rate decay. A model is then trained for each cross-validation split, on a shortlist of hyperparameter combinations, shown in Table 3. Each combination is executed 30 times and the model with the highest validation performance is used as the final model. Each set of four models is used as an ensemble predictor for the respective test partition. The logarithm of the probability distribution of each of the four models is averaged and used as an ensemble prediction. The web service predictions are generated with a 20-model ensemble. The neural network is trained with stochastic gradient descent using the Adam optimizer (Kingma and $\mathrm{Ba}, 2014)$. The models are implemented with the PyTorch deep learning framework (Paszke et al., 2017).

\subsubsection{Quantitative evaluation criteria}

To evaluate the discrimination between GPI-anchored and non GPIanchored proteins we use the Matthews Correlation Coefficient (MCC) and for $\omega$-site prediction evaluation we use the F1 score (Baldi et al., 2000). The F1 score is the harmonic mean of sensitivity (how many of the true cleavage sites are predicted correctly) and precision (how many of the predicted cleavage sites are true). Due to the dual nature of the problem, and the lack of experimental $\omega$-site evidence in the training set, a simple heuristic is devised. The heuristic is a composition of the two evaluation methods. The F1 score is calculated with a tolerance of two positions from the annotated $\omega$-site. We allow for this flexibility when calculating the F1 score as the training set contains mostly non-experimentally verified $\omega$-site samples, which are not as reliable as the experimentally verified. The MCC is weighed twice as important as the F1 score. We weigh the MCC more as we want to emphasize the GPI-anchoring discrimination over the $\omega$-site prediction performance. The model with the combination of hyperparameters that gives the best heuristics, on the validation partition, is chosen for each fold. This heuristic also controls when the model's parameters are stored as an early stopping approach. The self evaluation metric during training is the Cross Entropy Loss.

\subsubsection{Qualitative evaluation methods}

To visualize the decision making of the model, we perform a feature importance analysis using the Local Interpretable Modelagnostic Explanations (LIME) package (Ribeiro et al., 2016). We perform this analysis on each partition separately, using the corresponding four model ensemble. In the LIME analysis, amino acids contributing to a GPI-anchored prediction will have a positive importance, while amino acids contributing to the non GPI-anchored prediction will have a negative importance. The larger the weight, the larger the contribution to the prediction.

Furthermore, we investigate the sequence composition around the $\omega$-site to uncover possible model biases.

\section{Results and discussion}

\subsection{Quantitative results}

The subset of the GPI dataset that is used for benchmarking contains 160 GPI-anchoring signal sequence samples, regarded as positive, and 2573 samples without a GPI-anchoring signal sequence, regarded as negative. To benchmark the $\omega$-site position prediction the positive

Table 3

Four combinations of hyperparameters with the best validation performance, every model has 4 LSTM layers and is trained for 300 epochs.

\begin{tabular}{|c|c|c|c|c|c|c|c|}
\hline e.d. $(d)$ & LSTM h.u. $\left(d^{\prime}\right)$ & LSTM dropout & a.d. $\left(d^{\prime \prime}\right)$ & lr. & lr. decay & w. decay & batch size \\
\hline 16 & 20 & 0.62 & 340 & 0.002479 & 0.9970 & 0.001930 & 32 \\
\hline 16 & 16 & 0.55 & 283 & 0.003346 & 0.9987 & 0.009095 & 128 \\
\hline 16 & 16 & 0.6 & 283 & 0.003346 & 0.9987 & 0.010052 & 128 \\
\hline 22 & 22 & 0.6 & 283 & 0.003346 & 0.9987 & 0.009095 & 128 \\
\hline
\end{tabular}

Abbreviations: e.d. = embedding dimension, h.u. = hidden units, a.d. = attention dimension, lr. = learning-rate, w. = weight. 
set is limited to the 50 , out of the 160 positive protein samples, with an experimentally verified $\omega$-site annotation.

To benchmark the performance of the existing tools the dataset was submitted to the three tools currently available; Big-П, GPI-SOM, and PredGPI. In the case of Big-П we separated the benchmark set according to kingdom and submitted to the corresponding versions of the tool. Sequences not belonging to animals, plants, or fungi were submitted to the protozoan version. Big- $\Pi$ annotates its predictions according to likelihood. Predictions with high likelihood are labeled as $P$, twilight zone predictions are labeled as $S$, and non-potentially GPIanchored proteins are labeled as $N$. We regarded any protein predicted as potentially GPI-anchored ( $P$ or $S$ ) as a GPI-anchored prediction.

PredGPI ranks and classifies predictions according to specificity. Predictions are regarded as highly probable, probable, weakly probable, and non GPI-anchored. We measure the performance for two settings of PredGPI; designating weakly probable either as GPI-anchored or non GPI-anchored. Assuming weakly probable as negative predictions gives the best performance according to MCC, as shown in Table 4.

For predicting the presence of GPI-anchors, NetGPI achieves the highest MCC of $\mathbf{0 . 8 9 5}$. It also attains the highest true positive rate (TPR), 0.975, the second highest being GPI-SOM. NetGPI achieves the highest precision, 0.834, the second highest being Big- $\Pi$ with a precision of 0.830 . Big- $\Pi$ has the second highest MCC, 0.817 and the lowest false positive rate (FPR), 0.010, whereas NetGPI has the second lowest FPR, 0.012. For a detailed comparison see Table 4.

We find that the Big-П learning set has at least 58 samples which overlap with our positive benchmark set and an unknown overlap with our negative benchmark set, as the negative set is not reported. This might cause the performance of Big- $\Pi$ to be overestimated. The publishing date of Eisenhaber et al. (2004) is the 19th of March 2004, however the metazoa and protozoa predictors are reported to have been updated on the 17th of June 2005. We filter the benchmark set to GPI-positive samples not found in Big-П's reported training set and non GPI-anchored samples made available on UniProt after 2005-06-17. In the filtered comparison the performance gap between NetGPI and Big- $\Pi$ increases from 0.078 MCC to 0.141 MCC. All of Big-П's false negative predictions belong to the filtered dataset. If we regard PredGPI's weakly probable as negative, the second highest MCC is, on the filtered dataset, achieved by PredGPI, with an MCC of 0.813 .

For the prediction of the position of the $\omega$-site we only consider the 50 proteins with an experimentally verified $\omega$-site. The aforementioned dataset overlap is overly prevalent for these proteins, out of the $50 \omega$-sites, 33 are used for training the Big- $\Pi$ model.
NetGPI correctly predicts 32 out of the 50 experimentally verified $\omega$-sites, with an F1 score of 0.496 . NetGPI correctly predicts 8 out of the 17 not found in the reported Big- $\Pi$ training set, with an F1 score of 0.372 . Big- $\Pi$ correctly predicts $38 / 50$, with an F1 score of 0.628 and $8 / 17$, with an F1 score of 0.457 . GPI-SOM correctly predicts 9/17, however the F1 score is only 0.151 because of GPI-SOM's higher false positive rate. If we allow for a one-off error window around the true $\omega$-site, then NetGPI outperforms Big- $\Pi$, correctly predicting 44/50, with an F1 score of 0.682 and 13/17, with an F1 score of 0.605 . Big- $\Pi$ correctly predicts $41 / 50$, with an F1 score of 0.677 and $10 / 17$, with an F1 score of 0.555 . The $\omega$-site position prediction results are detailed in Table 5.

\subsection{Qualitative results}

In the qualitative analysis we investigate the importance of biological features when NetGPI predicts GPI-anchor presence and the $\omega$-site. In addition, we analyze the $\omega$-site composition to understand the neighborhood of true and predicted $\omega$-site positions. Furthermore, we investigate model likelihood of the predictions, and how it relates to model correctness. Lastly, we use NetGPI to predict GPI-anchored proteins in six complete proteomes.

\subsubsection{Feature Importance analysis}

Fig. 2 illustrates the results of the LIME analysis for both positive (see Fig. 2a) and negative (see Fig. 2b) samples. We observe that the presence of a hydrophobic tail contributes the most towards a positive prediction. This is consistent with the literature (Orlean and Menon, 2007), which defines the presence of a hydrophobic region from the position $\omega+10$. From that position the feature importance is much higher than for the rest of the sequence, which means that the main feature driving the positive prediction of NetGPI is the presence of the hydrophobic region. Regarding the negative predictions, we observe that the amino acids contributing the most towards a negative prediction are charged and polar amino acids. This indicates that the model is attributing higher importance to non-hydrophobic amino acids, indicating a lack of hydrophobic tail, when making a negative prediction.

\subsection{2. $\omega$-site composition}

Out of the 50 proteins with an experimentally verified $\omega$-site annotation there are 25 metazoa (animal) proteins, 13 plant proteins, 7 fungi proteins and 5 protozoa (other) proteins. Of the 25 animal proteins there are 14 which belong to Homo sapiens. All of the 13 plant proteins belong to the same species, Arabidopsis thaliana. Of the 50

Table 4

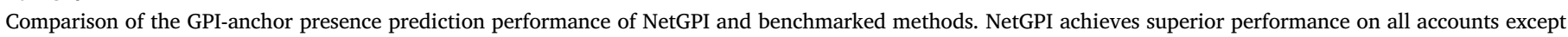
FPR where it is outperformed by Big-П.

\begin{tabular}{|c|c|c|c|c|c|c|c|c|}
\hline All (2733) & TP & FP & FN & $\mathrm{TN}$ & TPR & Prec. & FPR & MCC \\
\hline NetGPI & 156 & 31 & 4 & 2542 & 0.975 & 0.834 & 0.012 & 0.895 \\
\hline PredGPI & 147 & 50 & 13 & 2523 & 0.919 & 0.746 & 0.019 & 0.816 \\
\hline PredGPI $^{* *}$ & 150 & 119 & 10 & 2454 & 0.938 & 0.558 & 0.046 & 0.702 \\
\hline GPI-SOM & 152 & 259 & 8 & 2314 & 0.950 & 0.370 & 0.101 & 0.558 \\
\hline BigPI & 132 & 27 & 28 & 2546 & 0.825 & 0.830 & 0.010 & 0.817 \\
\hline Filtered $^{* * * *}(1080)$ & TP & FP & FN & $\mathrm{TN}$ & TPR & Prec. & FPR & MCC \\
\hline NetGPI & 98 & 11 & 4 & 967 & 0.961 & 0.899 & 0.011 & 0.922 \\
\hline PredGPI ${ }^{*}$ & 92 & 28 & 10 & 950 & 0.902 & 0.767 & 0.029 & 0.813 \\
\hline BigPI & 74 & 10 & 28 & 968 & 0.725 & 0.881 & 0.010 & 0.781 \\
\hline
\end{tabular}

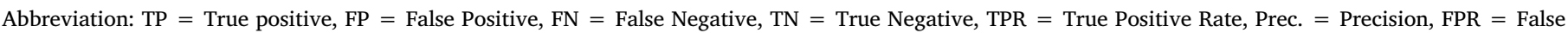
Positive Rate, MCC = Matthews Correlation Coefficient.

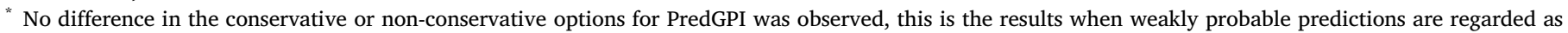
negative.

${ }_{* *}$ This is the result for PredGPI when weakly probable predictions are regarded as positive.

${ }^{* * *}$ Here the samples are limited to positive samples not in Big-П's reported training set and negative samples made available on UniProt after 2005-06-17 
Table 5

Comparison of the $\omega$-site position prediction performance of NetGPI and the benchmarked methods.

\begin{tabular}{|c|c|c|c|c|c|c|c|c|c|c|c|c|}
\hline Known $^{* * k * k}(50)$ & \pm 0 & F1 & Sens. & Prec. & \pm 1 & F1 & Sens. & Prec. & \pm 2 & $\mathrm{~F} 1$ & Sens. & Prec. \\
\hline NetGPI & 32 & 0.496 & 0.640 & 0.405 & 44 & 0.682 & 0.880 & 0.557 & 44 & 0.682 & 0.880 & 0.557 \\
\hline PredGPI* & 29 & 0.403 & 0.580 & 0.309 & 35 & 0.486 & 0.700 & 0.372 & 36 & 0.500 & 0.720 & 0.383 \\
\hline PredGPI & 28 & 0.389 & 0.560 & 0.298 & 36 & 0.500 & 0.720 & 0.383 & 37 & 0.514 & 0.740 & 0.394 \\
\hline PredGPI ${ }^{*, * *}$ & 29 & 0.272 & 0.580 & 0.178 & 35 & 0.329 & 0.700 & 0.215 & 36 & 0.338 & 0.720 & 0.221 \\
\hline PredGPI ${ }^{* *}$ & 28 & 0.263 & 0.560 & 0.172 & 36 & 0.338 & 0.720 & 0.221 & 37 & 0.347 & 0.740 & 0.227 \\
\hline GPI-SOM & 30 & 0.182 & 0.600 & 0.107 & 33 & 0.200 & 0.660 & 0.118 & 33 & 0.200 & 0.660 & 0.118 \\
\hline BigPI & 38 & 0.628 & 0.760 & 0.535 & 41 & 0.677 & 0.820 & 0.577 & 41 & 0.677 & 0.820 & 0.577 \\
\hline Known $^{\text {t*k***** }}(17)$ & \pm 0 & F1 & Sens. & Prec. & \pm 1 & F1 & Sens. & Prec. & \pm 2 & $\mathrm{~F} 1$ & Sens. & Prec. \\
\hline NetGPI & 8 & 0.372 & 0.471 & 0.308 & 13 & 0.605 & 0.765 & 0.500 & 13 & 0.605 & 0.765 & 0.500 \\
\hline PredGPI" & 5 & 0.172 & 0.294 & 0.122 & 9 & 0.311 & 0.529 & 0.220 & 10 & 0.345 & 0.588 & 0.244 \\
\hline PredGPI & 4 & 0.138 & 0.235 & 0.098 & 9 & 0.311 & 0.529 & 0.220 & 10 & 0.345 & 0.588 & 0.244 \\
\hline PredGPI ${ }^{*, * *}$ & 5 & 0.121 & 0.294 & 0.076 & 9 & 0.216 & 0.529 & 0.136 & 10 & 0.242 & 0.588 & 0.152 \\
\hline PredGPI ${ }^{* *}$ & 4 & 0.097 & 0.235 & 0.061 & 9 & 0.216 & 0.529 & 0.136 & 10 & 0.242 & 0.588 & 0.152 \\
\hline GPI-SOM & 9 & 0.152 & 0.529 & 0.089 & 10 & 0.169 & 0.588 & 0.099 & 10 & 0.169 & 0.588 & 0.099 \\
\hline BigPI & 8 & 0.457 & 0.421 & 0.500 & 10 & 0.555 & 0.588 & 0.526 & 10 & 0.555 & 0.588 & 0.526 \\
\hline
\end{tabular}

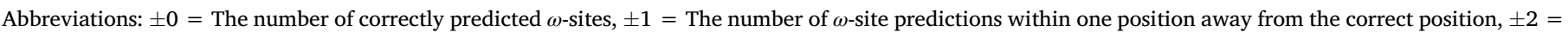
The number of $\omega$-site predictions within two positions away from the correct position, F1 = F1-score, Sens. = Sensitivity, Prec. $=$ Precision.

* PredGPI provides two options, this is their conservative option.

** This is the result for PredGPI when weakly probable predictions are regarded as positive.

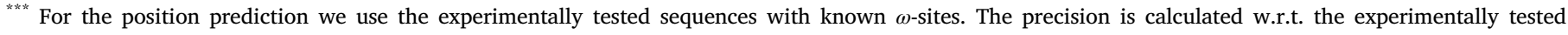
sequences with known $\omega$-sites as well as all negative samples.

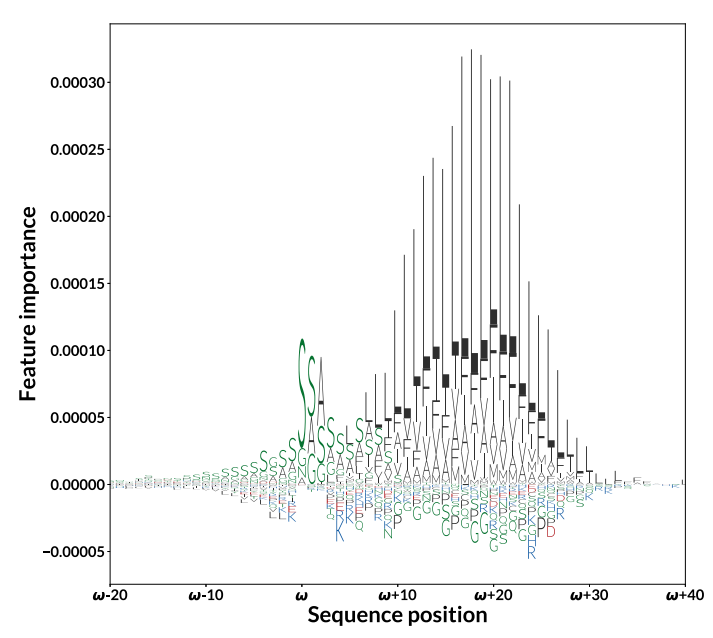

(a) Positive predictions

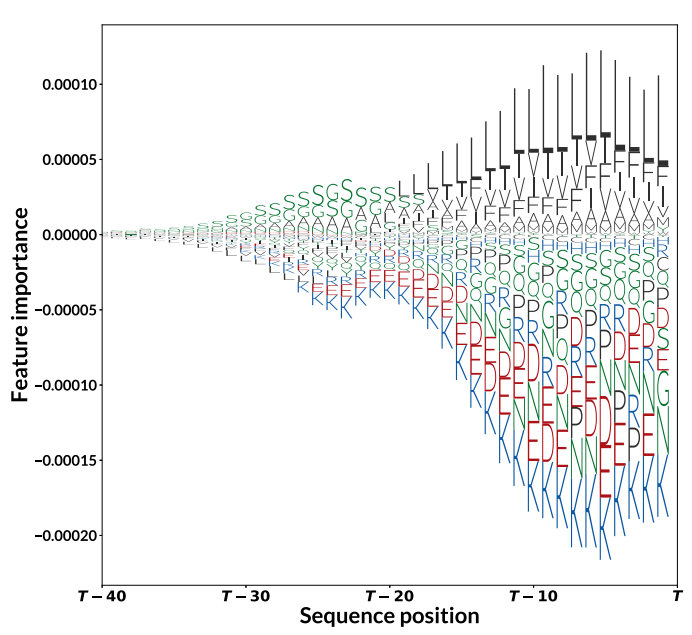

(b) Negative predictions

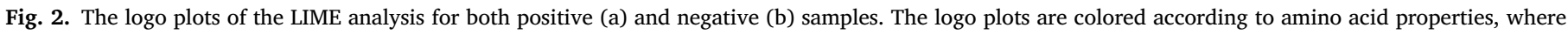

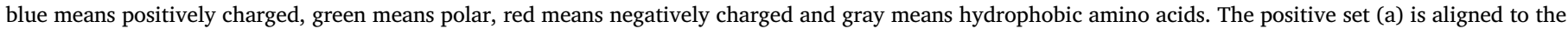

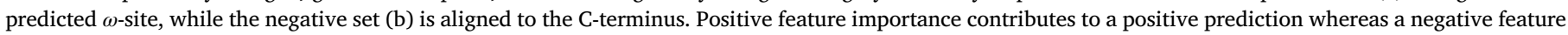

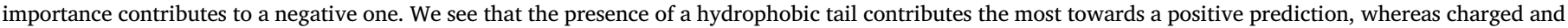

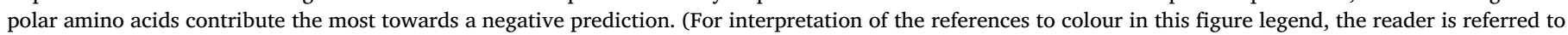
the web version of this article.)

experimentally verified $\omega$-sites, 27 are Serine, while the other amino acids observed are Asparagine, Glycine, Aspartic acid, Cysteine and Alanine, in decreasing order of frequency. The $\omega$-site of five of the Arabidopsis thaliana proteins are correctly positioned by NetGPI. The other eight are all one-off errors and constitute $\frac{2}{3}$ of one-off errors made by NetGPI. Out of those, there are seven where the $\omega$-site amino-acid is Serine (S) where the predicted amino-acid is the Aspartic Acid (D) in the $\omega+1$ position. All seven have in common the 4-mer $[\omega-2, \omega+1]$ motif PTSD, followed either by Glycine (G) or Alanine (A) in position $\omega+2$. Both Big- $\Pi$ and NetGPI are unable to position 3 out of 4 Aspartic acid $\omega$-sites. This may be related to the $\omega+2$ position, as these 3 samples have a non-standard amino acid (i.e. something other than G, A, or S). See Table 6.

\subsubsection{Likelihood and correctness}

In addition to the classification of the sequence and the most likely position of the $\omega$-site, NetGPI reports the likelihood of the chosen position. For positive predictions this is the predicted $\omega$-site, while for negative predictions it is the sentinel.

As our model is trained with cross entropy, it is penalized with a logarithm of the correct prediction. If we predict incorrectly, with a very low likelihood for the correct position, the loss can be immense. We should thus expect that answers with a high likelihood are more credible.

In Fig. 3 we display the likelihood distribution of the predictions. We observe differences in the likelihood of correct and incorrect predictions implying a correlation between likelihood and correctness. 
Table 6

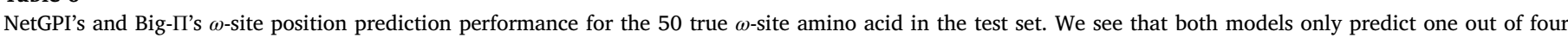

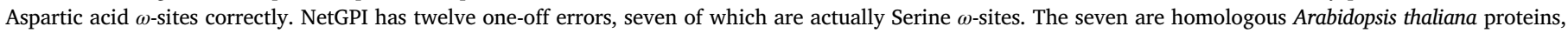
which have in common the tetramer $[\omega-2, \omega+1]$ motif PTSD, followed either by Glycine (G) or Alanine (A) in position $\omega+2$.

\begin{tabular}{|c|c|c|c|c|c|c|}
\hline NetGPI & S (27) & $\mathrm{N}(9)$ & G (6) & D (4) & C (2) & A (2) \\
\hline \pm 0 & 20 & 7 & 4 & 1 & 0 & 0 \\
\hline \pm 1 & 27 & 8 & 5 & 1 & 2 & 1 \\
\hline \pm 2 & 27 & 8 & 5 & 1 & 2 & 1 \\
\hline B1gPI & $s(27)$ & $N(9)$ & $G(6)$ & $D(4)$ & $C(2)$ & $A(2)$ \\
\hline \pm 0 & 21 & 9 & 4 & 1 & 2 & 1 \\
\hline \pm 1 & 23 & 9 & 5 & 1 & 2 & 1 \\
\hline \pm 2 & 23 & 9 & 5 & 1 & 2 & 1 \\
\hline
\end{tabular}

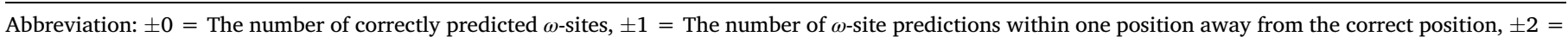
The number of $\omega$-site predictions within two positions away from the correct position.

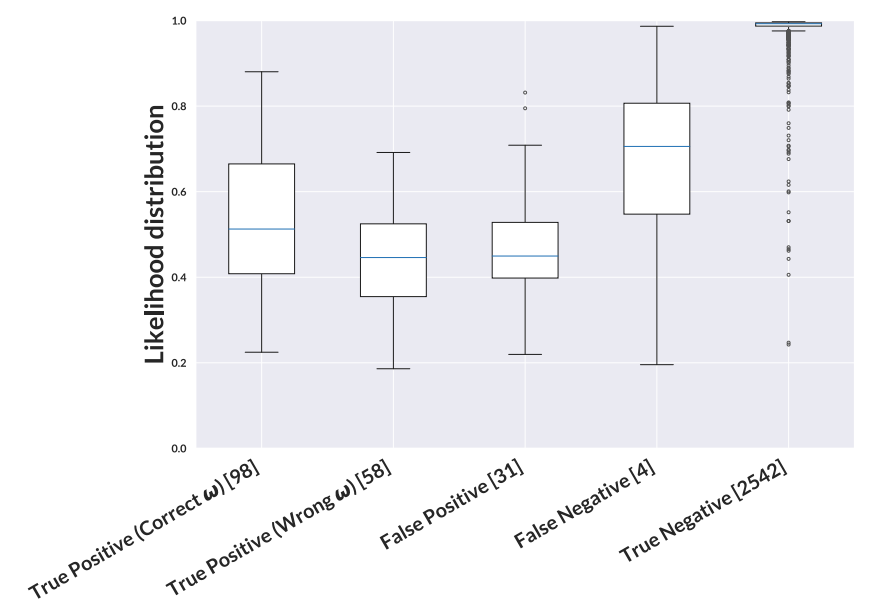

Fig. 3. The likelihood distribution for true positive, false positive, false negative and true negative predictions. True positive are split into correctly positioned $\omega$-sites and incorrectly positioned. The number of samples behind each are displayed in brackets.

Furthermore, we observe higher likelihood in negative predictions than positive. This is expected as the probability distribution covers the last 100 amino acids as well as the added sentinel. Only the sentinel position denotes a negative prediction, while a positive prediction is spread across the 100 amino acid positions. This means that positive prediction likelihood has to cover all potential $\omega$-site positions, while the negative prediction likelihood is limited to one position. Therefore, using the likelihood as ranking should be done separately for negative and positive results.

\subsubsection{Proteome analysis}

To further confirm the validity of NetGPI predictions, we predicted GPI-anchored proteins in six model organism proteomes: Arabidopsis thaliana, Drosophila melanogaster, Homo sapiens, Mus musculus, Try- panosoma brucei, and Saccharomyces cerevisiae. These proteomes are extracted from the UniProt database, release 2020_04 (UniProt Consortium, 2014). We ran the proteomes first through SignalP 5.0 (Armenteros et al., 2019) to detect proteins with a signal peptide. These proteins were then run through NetGPI to predict the presence of a GPI signal. Table 7 shows the results from the proteome analysis. We can observe that for most proteomes the percentage of predicted GPI-anchored proteins is close to $1 \%$. Trypanosoma brucei is the only organism with an elevated percentage of GPI-anchored proteins (2.17\%). This corresponds well with the known prevalence of GPIanchored variant surface glycoproteins in the trypanosomal genome (Ferguson, 1999).

\section{Conclusion}

We have shown that GPI-anchor prediction can be improved using recurrent neural networks and up-to-date datasets. Comparison with previous methods is challenging as there exists no standard dataset for training and testing predictive methods. Given progress in protein annotation, we publish a new homology partitioned dataset, using both experimentally verified proteins and manually annotated predicted proteins for training and validation. Due to the new dataset definition, the performance of current methods could be overestimated as their training sets contain sequences which are identical or homologous to sequences in our benchmark set.

Our results indicate that proteins manually annotated by prediction methods or sequence similarity are useful for training a GPI-anchor predictor to perform well when evaluated on experimentally verified GPI-anchoring signals. However, using these data may have increased the number of $\omega$-site predictions that are off by one position. We believe that this limitation is necessary in order to obtain a larger training set. If we were to use only the experimentally verified GPIanchors to train and test the predictor, we would not have enough training samples to teach a deep neural network classifier.

A web server implementing NetGPI is available at https://services. healthtech.dtu.dk/service.php?NetGPI. Our dataset can be downloaded from the same site or accessed in the supplementary materials.

Table 7

The number of GPI-anchored found by NetGPI in various proteomes.

\begin{tabular}{|c|c|c|c|c|}
\hline Proteome & \#Proteins & \#Proteins with signal peptide & \#Proteins with GPI & $\%$ with GPI \\
\hline A. thaliana & 27500 & 3675 & 278 & $1.01 \%$ \\
\hline D. melanogaster & 13811 & 3178 & 179 & $1.30 \%$ \\
\hline H. sapiens & 20600 & 3516 & 193 & $0.94 \%$ \\
\hline M. musculus & 21989 & 3940 & 210 & $0.96 \%$ \\
\hline T. brucei & 8561 & 652 & 186 & $2.17 \%$ \\
\hline S. cerevisiae & 6049 & 314 & 69 & $1.14 \%$ \\
\hline Total & 98510 & 15275 & 1115 & $1.13 \%$ \\
\hline
\end{tabular}




\section{CRediT authorship contribution statement}

Magnús Halldór Gíslason: Methodology, Investigation, Software, Data curation, Writing - original draft. Henrik Nielsen: Conceptualization, Supervision, Project administration, Writing - review \& editing. José Juan Almagro Armenteros: Methodology, Supervision, Writing - review \& editing. Alexander Rosenberg Johansen: Methodology, Supervision, Writing - review \& editing.

\section{Declaration of Competing Interest}

The authors declare that they have no known competing financial interests or personal relationships that could have appeared to influence the work reported in this paper.

\section{Appendix A. Supplementary material}

Supplementary data associated with this article can be found, in the online version, at https://doi.org/10.1016/j.crbiot.2021.01.001.

\section{References}

Almagro Armenteros, J.J., Tsirigos, K.D., Sonderby, C.K., Petersen, T.N., Winther, O., Brunak, S., von Heijne, G., Nielsen, H., 2019. SignalP 5.0 improves signal peptide predictions using deep neural networks. Nature Biotechnol. 37, 420-423. https:// www.nature.com/articles/s41587-019-0036-z, doi:10.1038/s41587-019-0036-z.

Baldi, P., Brunak, S., Chauvin, Y., Andersen, C.A., Nielsen, H., 2000. Assessing the accuracy of prediction algorithms for classification: an overview. Bioinformatics 16, $412-424$.

Brul, S., King, A., Van der Vaart, J., Chapman, J., Klis, F., Verrips, C., 1997. The incorporation of mannoproteins in the cell wall of S. cerevisiae and filamentous ascomycetes. Antonie van Leeuwenhoek 72, 229-237.

Clark, S., Hayes, P., 2019. SigOpt Web page. https://sigopt.com.

Dondoshansky, I., Wolf, Y., 2019. Blastclust. ftp://ftp.ncbi.nih.gov/blast/documents/ blastclust.html.

Eisenhaber, B., Bork, P., Eisenhaber, F., 1999. Prediction of potential GPI-modification sites in proprotein sequences. J. Molecular Biol. 292, 741-758. http:// www.sciencedirect.com/science/article/pii/S0022283699930693, doi:10.1006/ jmbi.1999.3069.

Eisenhaber, B., Wildpaner, M., Schultz, C.J., Borner, G.H., Dupree, P., Eisenhaber, F., 2003. Glycosylphosphatidylinositol lipid anchoring of plant proteins. Sensitive prediction from sequence- and genome-wide studies for Arabidopsis and rice. Plant Physiol 133, 1691-1701. https://doi.org/10.1104/pp.103.023580 https://www. ncbi.nlm.nih.gov/pmc/articles/PMC300724/.

Eisenhaber, B., Schneider, G., Wildpaner, M., Eisenhaber, F., 2004. A sensitive predictor for potential GPI lipid modification sites in fungal protein sequences and its application to genome-wide studies for Aspergillus nidulans, Candida albicans, Neurospora crassa, Saccharomyces cerevisiae and Schizosaccharomyces pombe. J. Molecular Biol. 337, 243-253. https://doi.org/10.1016/j.jmb.2004.01.025 http:// www.sciencedirect.com/science/article/pii/S002228360400083X.

Fankhauser, N., Mäser, P., 2005. Identification of GPI anchor attachment signals by a Kohonen self-organizing map. Bioinformatics 21, 1846-1852. https://doi.org/ 10.1093/bioinformatics/bti299.

Ferguson, M., 1999. The structure, biosynthesis and functions of glycosylphosphatidylinositol anchors, and the contributions of trypanosome research. J. Cell Sci. 112, 2799-2809.

Graves, A., 2012. Supervised sequence labelling. In: Supervised sequence labelling with recurrent neural networks. Springer, pp. 5-13.

Hobohm, U., Scharf, M., Schneider, R., Sander, C., 1992. Selection of representative protein data sets. Protein Sci. 1, 409-417.

Hochreiter, S., Schmidhuber, J., 1997. Long short-term memory. Neural Comput. 9, 1735-1780. https://doi.org/10.1162/neco.1997.9.8.1735.
Jurtz, V.I., Johansen, A.R., Nielsen, M., Almagro Armenteros, J.J., Nielsen, H., Sønderby, C.K., Winther, O., Sønderby, S.K., 2017. An introduction to deep learning on biological sequence data: examples and solutions. Bioinformatics 33, 3685-3690.

Kawagoe, K., Kitamura, D., Okabe, M., Taniuchi, I., Ikawa, M., Watanabe, T., Kinoshita, T., Takeda, J., 1996. Glycosylphosphatidylinositol-anchor-deficient mice: implications for clonal dominance of mutant cells in paroxysmal nocturnal hemoglobinuria. Blood 87, 3600-3606.

Kingma, D.P., Ba, J., 2014. Adam: A method for stochastic optimization. arXiv [cs] abs/ 1412.6980. http://arxiv.org/abs/1412.6980.

Kinoshita, T., 2020. Biosynthesis and biology of mammalian GPI-anchored proteins. Open Biol. 10. 190290.

LeCun, Y., Bengio, Y., Hinton, G., 2015. Deep learning. Nature 521, 436-444.

Li, W., Godzik, A., 2006. Cd-hit: a fast program for clustering and comparing large sets of protein or nucleotide sequences. Bioinformatics 22, 1658-1659.

Masterson, W.J., Raper, J., Doering, T.L., Hart, G.W., Englund, P.T., 1990. Fatty acid remodeling: a novel reaction sequence in the biosynthesis of trypanosome glycosyl phosphatidylinositol membrane anchors. Cell 62, 73-80.

Mayor, S., Riezman, H., 2004. Sorting GPI-anchored proteins. Nature Rev. Molecular Cell Biol. 5, 110.

McCann, B., Keskar, N.S., Xiong, C., Socher, R., 2018. The natural language decathlon: Multitask learning as question answering. arXiv [cs, stat] abs/1806.08730. http:// arxiv.org/abs/1806.08730, arXiv:1806.08730.

Merity, S., Xiong, C., Bradbury, J., Socher, R., 2016. Pointer sentinel mixture models. arXiv [cs] abs/1609.07843. http://arxiv.org/abs/1609.07843, arXiv:1609.07843.

Mikolov, T., Sutskever, I., Chen, K., Corrado, G., Dean, J., 2013. Distributed representations of words and phrases and their compositionality. In: Proceedings of the 26th International Conference on Neural Information Processing Systems Volume 2, Curran Associates Inc., USA. pp. 3111-3119. http://dl.acm.org/citation. cfm?id $=2999792.2999959$.

Needleman, S.B., Wunsch, C.D., 1970. A general method applicable to the search for similarities in the amino acid sequence of two proteins. J. Mol. Biol. 48, 443-453. http://www.sciencedirect.com/science/article/pii/0022283670900574, doi: 10.1016/0022-2836(70)90057-4.

Orlean, P., Menon, A.K., 2007. GPI anchoring of protein in yeast and mammalian cells, or: how we learned to stop worrying and love glycophospholipids. J. Lipid. Res. 48, 993-1011.

Paszke, A., Gross, S., Chintala, S., Chanan, G., Yang, E., DeVito, Z., Lin, Z., Desmaison, A., Antiga, L., Lerer, A., 2017. Automatic differentiation in PyTorch. In: NIPS Autodiff Workshop. https://openreview.net/forum?id=BJJsrmfCZ\&noteId = BJJsrmfCZ.

Pearson, W.R., Lipman, D.J., 1988. Improved tools for biological sequence comparison. Proc. Natl. Acad. Sci. USA 85, 2444-2448. doi:10.1073/pnas.85.8.2444.

Pierleoni, A., Martelli, P.L., Casadio, R., 2008. PredGPI: a GPI-anchor predictor. BMC Bioinformatics 9, 392. https://www.ncbi.nlm.nih.gov/pmc/articles/PMC2571997/ , doi:10.1186/1471-2105-9-392.

Poisson, G., Chauve, C., Chen, X., Bergeron, A., 2007. FragAnchor: a large-scale predictor of glycosylphosphatidylinositol anchors in eukaryote protein sequences by qualitative scoring. Genomics Proteomics Bioinformatics 5, 121-130. https://doi. org/10.1016/S1672-0229(07)60022-9 https://pubmed.ncbi.nlm.nih.gov/ 17893077.

Ribeiro, M.T., Singh, S., Guestrin, C., 2016. Why should I trust you?: Explaining the predictions of any classifier. arXiv [cs, stat] abs/1602.04938. http://arxiv.org/abs/ 1602.04938, arXiv:1602.04938.

Schuster, M., Paliwal, K., 1997. Bidirectional recurrent neural networks. Trans. Sig. Proc. 45, 2673-2681. https://doi.org/10.1109/78.650093.

Steinegger, M., Söding, J., 2017. MMseqs2 enables sensitive protein sequence searching for the analysis of massive data sets. Nature Biotechnol 35, 1026-1028. https://doi. org/10.1038/nbt.3988.

Takeda, J., Miyata, T., Kawagoe, K., Iida, Y., Endo, Y., Fujita, T., Takahashi, M., Kitani, T., Kinoshita, T., 1993. Deficiency of the GPI anchor caused by a somatic mutation of the PIG-A gene in paroxysmal nocturnal hemoglobinuria. Cell 73, 703-711.

UniProt Consortium, 2014. UniProt: a hub for protein information. Nucl. Acids Res. 43, D204-D212.

Vinyals, O., Fortunato, M., Jaitly, N., 2015. Pointer networks. arXiv [cs, stat] abs/ 1506.03134. http://arxiv.org/abs/1506.03134, arXiv:1506.03134.

Werbos, P.J., 1990. Backpropagation through time: what it does and how to do it. Proc. IEEE 78, 1550-1560. 